\title{
La formation des enseignants dans les Balkans Occidentaux
}

Teacher training in the Western Balkans

La formación de los profesores en los Balcanes Occidentales

\section{Pavel Zgaga}

\section{(2) OpenEdition}

\section{Journals}

Édition électronique

URL : http://journals.openedition.org/ries/968

DOI : $10.4000 /$ ries.968

ISSN : 2261-4265

Éditeur

Centre international d'études pédagogiques

Édition imprimée

Date de publication : 1 décembre 2010

Pagination : 129-140

ISBN : 978-2-8542-583-1

ISSN : $1254-4590$

\section{Référence électronique}

Pavel Zgaga, "La formation des enseignants dans les Balkans Occidentaux », Revue internationale d'éducation de Sèvres [En ligne], 55 | décembre 2010, mis en ligne le 01 décembre 2013, consulté le 21 avril 2019. URL : http://journals.openedition.org/ries/968 ; DOI : 10.4000/ries.968 


\section{La formation des enseignants dans les Balkans Occidentaux*}

\section{Pavel Zgaga}

Traiter de la formation des enseignants dans l'Europe du Sud-Est n'est pas une chose facile en raison du contexte particulier de cette région. Aujourd'hui, le terme de "Balkans Occidentaux » fait généralement référence à la formule suivante : ex-Yougoslavie - Slovénie + Albanie. Il y a vingt ans, cette formule était très différente. À l'exception de l'Albanie, les pays des Balkans Occidentaux sont associés à une part très importante de l'histoire politique du $\mathrm{XX}^{\mathrm{e}}$ siècle ainsi qu'à l'héritage d'un État commun, la Yougoslavie. Ses racines n'ont commencé à pousser qu'après la Première Guerre mondiale. À l'issue de la Seconde Guerre mondiale, la Yougoslavie fédérale socialiste, qui ne se situait ni à l'Est ni à l'Ouest, était plutôt décentralisée puis, vers le milieu des années soixante, elle s'est rapprochée de l'Ouest. Au sud-ouest de la péninsule, l'Albanie est restée volontairement isolée jusque dans les années quatre-vingt-dix. Considérant les Balkans dans un cadre plus large, la Bulgarie et la Roumanie appartenaient au bloc soviétique, alors que la Moldavie faisait partie intégrante de l'Union soviétique. Au sud des Balkans, la Grèce constituait, politiquement parlant, la frontière vers l'ouest.

On peut aisément comprendre que des contextes aussi variés aient également influencé la grande diversité que l'on observe dans les systèmes éducatifs de la région. Cependant, si l'on regroupe ces pays sous l'étiquette commune de "communistes ", on perd du même coup la plupart des éléments indispensables à la compréhension de la transition et de la reconstruction qui se sont déroulées au cours des vingt dernières années. Entre 1945 et 1990, trois systèmes politiques différents - et périodiquement même hostiles - se sont affrontés dans cette région : les pays du bloc soviétique, la Yougoslavie "nonalignée " et l'Albanie " autarcique ». Après 1990, l'empire soviétique a cessé d'exister. La Roumanie et la Bulgarie ont initié des changements et ont rejoint l'Union européenne en 2007. Après avoir surmonté de graves désordres dans les années 1990, l'Albanie a lancé des réformes politiques et économiques et s'est ouverte à l'Europe et au monde. La Yougoslavie, "la plus joyeuse des casernes " après la Seconde Guerre mondiale, s'est disloquée et nombre de ses morceaux

* Article traduit par Robert Elbaz. 
ont été dévorés par les flammes puis réduits en cendres dans les années quatre-vingt-dix. Il est impossible de débattre du rôle de l'éducation et des enseignants dans la récente reconstruction sociale qui s'est opérée dans la région sans prendre en compte ces différents contextes.

Un autre problème surgit lorsque l'on envisage d'étudier la formation des enseignants dans la région : l'absence de recherche systémique comparative. De ce point de vue, les circonstances qui ont précédé et qui ont prévalu au cours des années quatre-vingt-dix ont été fort peu propices. Le soi-disant Enhanced Graz Process $^{1}$ (Zgaga, 2005) fut un tournant: l'aide systémique internationale apportée pour renouveler et reconstruire les systèmes éducatifs (Gaber, 2000) a également fourni des occasions de développement pour les chercheurs en éducation. Toutefois, nombre de projets se sont concentrés principalement sur les systèmes généraux d'éducation, sans mettre l'accent sur la formation des maîtres. De plus, les traumatismes liés à la période d'après-guerre ont constitué un facteur aggravant qui a entravé la coopération régionale directe. Il y a eu en effet des études internes aux différents pays, mais guère d'enquêtes transnationales. La première enquête comparative à s'être intéressée exclusivement à la formation des maîtres n'a été conduite qu'en 2005-2006 (Zgaga, 2006). Un groupe de

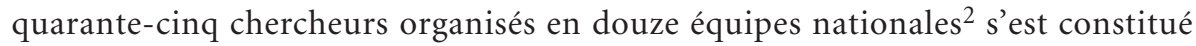
autour d'un projet unique (financé par le Open Society Institute et la Suisse) afin d'établir un bilan de la situation en matière de formation des maîtres, d'identifier les besoins des enseignants en exercice et d'envisager les perspectives pour l'avenir.

Deux ans plus tard, le tout récent Centre for Education Policy (Centre pour les politiques éducatives) de Belgrade a publié un rapport plutôt spécialisé portant sur les programmes de formation des maîtres dans les Balkans Occidentaux. Il cherchait également à renforcer « la coopération régionale dans le processus d'introduction et de développement de programmes de formation des maîtres fondés sur les compétences» (Pantić, 2008). Cet objectif a été atteint : des chercheurs de cinq pays de l'ex-Yougoslavie ont publié un excellent rapport comparatif, Regional Tuning ${ }^{3}$. Très récemment, le troisième projet comparatif régional centré sur le développement des compétences en matière d'éducation inclusive ${ }^{4}$ dans les Balkans Occidentaux a également été mené à bien (Scienter et CEP, 2010).

\footnotetext{
1. Processus élargi de $\mathrm{Graz}(\mathrm{NdT})$.

2. Les équipes nationales couvraient une région large et extrêmement diverse, bordée par l'Albanie et la Macédoine au sud, la Moldavie à l'est et la Slovénie et l'Autriche (pour des raisons comparatives) au nord.

3. Le projet reposait sur une méthodologie mise au point dans le cadre d'un projet européen très connu, Tuning, conduit par l'université Deusto (Espagne) et l'Université de Groningue (Pays-Bas), qui visait à encourager la mise en place du Processus de Bologne dans les pays des Balkans Occidentaux (NdA). Le mot «tuning » évoque l'idée d'un réglage fin, d'une mise au point, en particulier celle d'une radio ou d'un moteur (NdT). 4. Enseignement des personnes à besoins éducatifs particuliers (EURYDICE/TESE).
} 
Dans les pages suivantes, quelques résultats essentiels provenant du premier projet (2006) sont présentés. Les données ont été collectées sous forme de questionnaires auxquels ont répondu 131 institutions concernées par la formation des maîtres dans onze pays et par 2290 enseignants en exercice dans douze pays. Les résultats de cette étude ont été analysés et débattus dans le cadre du groupe de recherches.

\section{LES INSTITUTIONS CONCERNÉES PAR LA FORMATION DES MAÎTRES}

Le processus d' " universitarisation ${ }^{5}$ de la formation initiale des enseignants, c'est-à-dire la transition que connaît cette formation, passant de la licence au master - tendance qui a émergé dans de nombreux pays européens dans les années quatre-vingt -, est également pratiquement achevé dans les pays de l'Europe du Sud-Est. Presque toutes les institutions ayant participé à l'étude sont des institutions d'enseignement supérieur assurant une formation des enseignants tout à la fois avant leur prise de fonctions puis sur le terrain. Les soi-disant "teacher academies" (dispensant une formation universitaire en deux ans, une forme dominante de formation des maîtres au cours des décennies précédentes) ne représentent aujourd'hui qu'une infime minorité et sont en voie de disparition. Seuls les départements d'université - qu'il s'agisse de facultés spécialisées en formation pédagogique ou de facultés liées à une discipline, qui forment également des enseignants - et ces "teacher academies » peuvent légalement délivrer des qualifications d'enseignant. Il existe également dans tous les pays de la région des institutions spécialisées se concentrant exclusivement sur la formation des enseignants sur le terrain.

Il n'est pas facile de décrire l'institution type, de taille moyenne, de la région. Si l'on ne considère que les étudiants du premier cycle, il y a pratiquement la même proportion de petites institutions allant jusqu'à cinq cents étudiants que de grandes, qui dépassent les deux mille. Les très petites et les très grandes institutions représentent presque les deux tiers du total. Cependant, ces caractéristiques ne s'appliquent pas lorsque nous observons les personnels d'enseignement et d'administration: presque la moitié des institutions n'emploient que trente enseignants et jusqu'à dix personnels administratifs, alors que moins d'un cinquième d'entre elles déclarent disposer respectivement de plus de 151 enseignants et de plus de 51 personnels administratifs. Ces chiffres montrent qu'il existe très probablement une sérieuse disproportion entre le niveau de recrutement des étudiants, qui ne cesse de monter, et le financement limité des institutions, qui provoque en toute logique un manque de ressources humaines.

5. L'auteur utilise le néologisme « universitisation » (NdT). 
Les tendances dans le nombre d'étudiants du second cycle (master et doctorat) diffèrent de celles que l'on observe dans le premier cycle : près de la moitié des institutions admettent jusqu'à cinquante étudiants diplômés, suivies par un cinquième qui en admet entre 51 et 100 et un autre cinquième allant de 101 à 500 étudiants. Le nombre total d'institutions qui assurent les programmes de cours de master et de doctorat est inférieur au nombre de celles qui n'assurent qu'un programme de premier cycle. Mais il est encore nécessaire de promouvoir les études tout à la fois au niveau du master et du doctorat ; cependant, une grande majorité d'institutions d'enseignement supérieur dans l'Europe du Sud-Est assure cet enseignement dans le domaine de la formation des maîtres.

L'étude a brossé un tableau optimiste en ce qui concerne la coopération entre les institutions chargées de la formation des maîtres et les établissements : une majorité absolue $(88,6 \%)$ des institutions a fait état d'une telle coopération. Celle-ci a pour but principal de fournir une ouverture pratique à l'enseignement théorique des étudiants de premier cycle ainsi que d'informer et de promouvoir l'offre de ces institutions sur le terrain, en direction des enseignants en exercice. Il est intéressant de remarquer que presque la moitié des participants à l'étude considère que l'intérêt dont les institutions font preuve en coopérant avec les établissements s'explique par la nécessité d'avoir accès à un environnement adéquat pour leurs propres projets de recherche et de développement, et d'inciter les enseignants à prendre part à ces activités. Cependant, seule une institution sur dix considère que la coopération avec les établissements est importante pour offrir des débouchés à leurs diplômés.

En dépit des accents quelque peu optimistes du paragraphe précédent, les projets de recherche et de développement ne sont pas la caractéristique la plus saillante pour une majorité d'institutions : moins de la moitié ont déclaré que ces projets - menés dans le champ de la formation des maîtres constituaient pour eux une activité régulière. Pour moins d'un tiers d'entre elles, ils faisaient occasionnellement partie de leurs activités. Près d'un quart ne mènent aucune recherche et considèrent que leur mission principale réside dans l'éducation et la formation. La publication universitaire n'est pas mieux lotie: seule une petite moitié des institutions ayant participé à l'étude ont déclaré que la publication faisait régulièrement partie de leurs activités. Dans la plupart des États de la région, les technologies de l'information et des communications en matière de formation des maîtres en sont toujours à leurs balbutiements. Moins d'un cinquième des institutions utilise systématiquement les TIC dans leur formation initiale ou sur le terrain, alors que la majorité n'a commencé à utiliser les TIC que dans certaines zones, tout en prévoyant d'étendre ces activités à l'avenir. Cette zone n'est pas délimitée de façon significative et manque tout à la fois de ressources financières et de ressources humaines. 
Une partie significative de l'étude s'est concentrée sur la formation initiale des maîtres, en particulier sur les activités menées en matière de réforme des programmes. Par le passé, nous avions déjà conduit une semblable étude (Zgaga, 2003), ce qui a permis de comparer certaines réponses. Bien entendu, le contexte a considérablement changé depuis 2003, et même depuis 2006. À l'époque de la première étude, de nombreux systèmes éducatifs de la région ne faisaient pas encore partie du Processus de Bologne. Cependant, en à peine trois ans, la prise de conscience du caractère nécessaire et même urgent des réformes structurelles ainsi que de la mise en conformité avec les directives européennes - dans l'enseignement supérieur en général et dans la formation des maîtres en particulier - s'est accrue de manière significative dans la région. Une étude ultérieure (Pantić, 2008) a montré tout à la fois un progrès et un échec dans la réforme des programmes menés dans le cadre de la mise en place du Processus de Bologne.

En 2006, moins de la moitié des participants à l'étude pensaient que leurs programmes faisaient preuve de qualité et d'efficacité ; d'autre part, ils pensaient qu'il était temps de préparer une réforme des programmes complète mais progressive pour promouvoir plus avant le renouvellement des systèmes nationaux et pour améliorer la compatibilité avec les directives européennes. La volonté générale de mettre en place des réformes de type Bologne était généralement très forte. Seule une infime minorité des participants considérait que leurs programmes étaient tout à fait modernes, de qualité et qu'ils s'articulaient bien aux besoins de la société. Ils considéraient qu'une réforme radicale ne s'imposait pas. Une autre infime minorité considérait, elle, que ses programmes étaient obsolètes et pensait qu'une réforme radicale était nécessaire et urgente. D'un autre côté, lorsque l'on considère les mesures concrètes qui ont été prises en matière de politique éducative, il semble que ce haut niveau de prise de conscience quant à la nécessité d'entreprendre des réformes au niveau institutionnel ne se matérialisait que dans des actions relativement mineures et fragmentées. En effet, les activités concrètes ont été plutôt parcellaires et l'on observe bon nombre de cas de colmatage et d'enjolivement superficiel en lieu et place d'une véritable planification de changements stratégiques.

Une question centrale - et débattue de manière très vive dans la région était la suivante : «quel modèle de structure diplômante fondée sur deux cycles avez-vous ou avez-vous l'intention d'adopter dans votre institution? » Bien que les institutions concernées par la formation des maîtres aient fait preuve d'une prise de conscience relativement aiguë de l'importance du problème des contenus à l'intérieur même de l'ordre du jour de la réforme, la question qui a émergé dans pratiquement tous les environnements nationaux était une question relativement "formelle": quelle devrait être la durée standard des programmes d'études précédant le diplôme ? Traduit dans notre jargon, on faisait référence à 
la formule: « $3+2 »$ ou « $4+1 »$ ? Dans le cadre de l'étude, les participants devaient choisir n'importe quelle variante possible. Il est intéressant de remarquer qu'un nombre presque égal de réponses se sont centrées autour de deux variantes principales : $32,3 \%$ des institutions ont opté pour un programme de premier cycle en quatre ans (bachelor/licence) suivi par un second cycle en un an (master) alors que $30,6 \%$ des institutions ont opté pour un premier cycle en trois ans suivi par un second cycle en deux ans. Ce résultat n'a pas vraiment constitué une surprise. Un résultat pratiquement identique avait été obtenu lors de notre première étude (Zgaga, 2003). D’autre part, en 2006, environ un cinquième des institutions envisageait encore les deux options et considérait souvent que l'une ou l'autre était mieux adaptée à tel ou tel profil donné. Seule une minorité d'entre elles a fait savoir qu'elle envisageait de ne gérer que le premier cycle (bachelor/licence ou équivalent). Pour autant que l'on puisse en juger aujourd'hui, la situation est encore très variée. Les changements de programmes sont récents et une nouvelle étude serait nécessaire pour présenter en détail les systèmes réformés.

Les réponses des institutions ont montré que deux approches modernes ont été utilisées de façon croissante dans le processus de restructuration des programmes. Plus de la moitié d'entre elles sont engagées de façon active dans la planification ou la mise au point de programmes fondés sur les résultats de l'apprentissage ${ }^{6}$; plus d'un quart d'entre elles déclarent que leurs programmes actuels sont déjà fondés sur ces résultats et/ou ces compétences. Lorsque l’on demande à ces mêmes institutions quels résultats sont - ou seront - mis au premier plan dans les nouveaux programmes, les connaissances fondamentales de la profession d'enseignant et les capacités à mettre en pratique les connaissances sont placés en tête de liste (environ pour les deux tiers d'entre elles), suivies par la maîtrise du sujet enseigné (pour la moitié d'entre elles).

Toutefois, la grande majorité des institutions choisissent encore la plupart du temps de recourir aux examens traditionnels comme format standard de leurs modalités d'évaluation des compétences définies, auxquels s'ajoutent des comptes rendus de travaux pratiques et des essais (pour les deux tiers d'entre elles), des projets et des épreuves pratiques (pour la moitié d'entre elles), des dossiers et des travaux de recherche (pour un quart d'entre elles). Les deux tiers des institutions utilisent déjà des systèmes de crédits, la plupart du temps des ECTS ${ }^{7}$. Seules quelques institutions ont fait état d'une "dérive populaire " de l'utilisation de ces crédits, comme l'attribution sur la base d'heures de présence dans un enseignement donné ou en fonction du statut et du prestige d'un enseignant.

Enfin, ces institutions devaient répondre à une question concernant les obstacles principaux à la réforme et la modernisation de la formation initiale 
des enseignants. Les participants devaient classer les options figurant dans le questionnaire sur une échelle allant de un (obstacle très important) à cinq (obstacle relativement facile à surmonter). Les notes moyennes sont les suivantes :

\begin{tabular}{|c|l|}
\hline 2,29 & Manque de support financier, concernant en particulier le matériel et les installations \\
\hline 2,79 & Réglementations nationales inappropriées \\
\hline 3,02 & $\begin{array}{l}\text { Manque de ressources humaines, de compétences adéquates et de motivation de la part } \\
\text { des personnels }\end{array}$ \\
\hline 3,32 & Manque de possibilités de coopération internationale dans la mise au point des programmes \\
\hline 3,42 & Manque d'exemples de bonnes pratiques, nationalement et internationalement \\
\hline
\end{tabular}

\section{Point de vue des enseignants EN EXERCICE SUR LA FORMATION DES MAÎTRES}

Outre les institutions concernées par la formation des enseignants, des professeurs en exercice ont également été consultés au sujet de leur formation initiale et de leur pratique sur le terrain, à la lumière de leur expérience passée comme à celle de leurs besoins actuels et futurs. En ce qui concerne le chapitre sur les institutions, cela ne présentait auparavant aucun intérêt de fournir une analyse comparative entre pays (avec seulement deux institutions dans le plus petit des pays, l'échantillon était bien trop réduit). Mais maintenant, cela était possible et nécessaire (avec cent trente-deux participants dans le plus petit pays).

On a demandé aux enseignants en exercice quel jugement ils portaient aujourd'hui sur leur formation initiale. En moyenne, plus de la moitié d'entre eux considérait qu'elle était suffisamment adaptée pour commencer à travailler sur le terrain, tout en éprouvant le besoin de davantage d'expériences pratiques d'enseignement, de formation et de suivi sur le terrain (nous appelons ces enseignants le groupe "en progrès»). Un tiers d'entre eux étaient encore plus enthousiastes au sujet de leur formation initiale, la trouvant adaptée et correspondant bien aux exigences de leur activité professionnelle. Fondamentalement, ils n'éprouvent pas le besoin de compléter leur formation et leur entraînement et forment le groupe "heureux». En moyenne, les réactions critiques ont été peu nombreuses. Moins d'un dixième d'entre eux ont trouvé que leur formation initiale était inadaptée, considérant que leur formation théorique ne correspondait guère aux exigences de leur activité professionnelle et que leur travail sur le terrain se fondait essentiellement sur des expériences personnelles et pratiques de l'enseignement, et sur la formation continue sur le terrain. Ils forment le groupe « critique».

Le tableau est différent si on l'observe depuis la perspective de chaque pays pris isolément. Le groupe "en progrès » est de loin le plus important en 
Autriche $(87,4 \%)$, suivi par la Serbie $(62,9 \%)$, l'Albanie $(61,4 \%)$ et la Croatie (62\%), alors qu'il est le plus petit en Moldavie (42,4\%) et au Monténégro $(43,8 \%)$. Le groupe «heureux» est le mieux représenté en Moldavie (53,0 \%) et au Monténégro (55,4\%), alors qu'il plafonne en Autriche (5,6\%), suivi d'assez loin par la Croatie (21,9\%), la Serbie (25,0 \%) et le Kosovo (25,8 \%). Le groupe «critique» est le plus développé en Croatie (16,9\%), suivi par la Bosnie-Herzégovine (13,3\%), la Roumanie (12,9\%) et la Serbie (12,1\%). Il est le plus réduit en Bulgarie (1,8\%), suivi par la Macédoine (3,0\%) et la Moldavie (3,3\%).

Il est difficile de donner une interprétation simple de ces résultats. Selon toute vraisemblance, cette interprétation devrait cependant prendre en compte au moins trois dimensions : la qualité, dans le passé comme aujourd'hui, de la formation initiale aussi bien que de la formation sur le terrain; le statut des enseignants et de leur motivation professionnelle (y compris les systèmes de salaires et de promotion) ; enfin, et tout autant, leur " potentiel critique».

L'étude est entrée davantage dans les détails lorsque l'on a demandé aux enseignants en exercice ce qu'ils pensaient des systèmes de formation pédagogique initiale dans leur pays et comment ils réagissaient aux défis posés par les réformes et aux promesses en matière d'enseignement supérieur européen aujourd'hui. Comme nous l'avons vu ci-dessus, les institutions de formation pédagogique ont émis un avis majoritairement positif sur le système et sur ses performances. Il en va de même avec les enseignants en exercice, dont plus de la moitié n'ont pas de critique à formuler sur les programmes et les structures existants. Cependant, tous ont considéré qu'il manquait à tout cela un composant spécifiquement "pédagogique ». À l'intérieur de ce groupe, la réponse la plus répandue a été que le programme d'études devrait mettre davantage l'accent sur les contenus, les sujets et les compétences pédagogiques spécialisés tels que l'enseignement, l'apprentissage, l'évaluation et la communication, ainsi que sur les expériences pratiques en relation avec les contenus, les sujets et les compétences théoriques. Il y a deux ans, la précédente étude était parvenue aux mêmes conclusions (Pantić, 2008).

Selon l'étude de 2006, les enseignants en exercice dans la région étaient très intéressés par le fait de pouvoir bénéficier d'une formation additionnelle. Seul un dixième d'entre eux ont déclaré qu'ils étaient tout à fait satisfaits de leur diplôme et de leur formation et qu'ils ne comptaient pas s'engager dans une poursuite d'études. Cependant, ils étaient beaucoup moins unanimes en ce qui concerne la formation additionnelle qui devrait suivre. Tout en haut, on observe deux groupes relativement différents, de tailles égales, représentant chacun environ un quart des réponses totales: quelques-uns entreprendraient des études de master, si possible dans leur matière d'enseignement ou dans leur champ pédagogique ; mais d'autre part, certains d'entre eux n'envisageraient pas de pousser leurs études plus avant et préféreraient disposer de davantage de 
formation sur le terrain. Bien sûr, il y avait des disparités assez significatives entre les pays. Par exemple, pas moins de la moitié des enseignants de BosnieHerzégovine entreprendraient des études de master alors que près d'un quart des enseignants slovènes et croates préféreraient davantage de formation sur le terrain.

Ces résultats requièrent une interprétation globale extensive. Plusieurs facteurs - tous n'étant pas également importants dans chaque pays - devraient être pris en compte. D'une part, les ambitions des enseignants, ainsi que leurs hésitations en ce qui concerne la formation additionnelle, sont déterminées de façon importante par les caractéristiques spécifiques de leurs systèmes nationaux de qualification (la définition de ces qualifications varie considérablement selon les pays). De l'autre, il semble que leur statut (et en particulier leur salaire) et leurs perspectives professionnelles constituent le second critère fortement déterminant. Il faudrait également prendre en compte les contextes culturels, par exemple le poids et la perception symbolique des diplômes universitaires, mais également les possibilités de passer d'un poste dans l'enseignement prétertiaire à un poste dans l'enseignement supérieur et la recherche ou dans des institutions publiques chargées du contrôle et du développement en matière d'éducation.

Selon le point de vue des enseignants en exercice, la formation sur le terrain semble même plus importante que la formation initiale. Ainsi, on a demandé aux enseignants d'évaluer ces systèmes de formation sur le terrain dans leur pays. Une large majorité, constituée de plus des deux tiers des participants, pense que des changements plus ou moins substantiels sont nécessaires. Plus d'un tiers considère que les programmes de formation sur le terrain devraient être élargis et comprendre des contenus et des sujets qui ne sont pas représentés aujourd'hui, alors qu'un autre tiers considère, lui, que l'offre et la qualité de cette formation sur le terrain devraient être améliorées de manière significative. Ces deux sous-groupes sont d'accord pour dire que l'offre devrait être mieux financée à partir de fonds publics. Si l'on rassemble ces deux groupes, ces déclarations font pratiquement figure de "consensus national » parmi les enseignants en Autriche et représentent une majorité écrasante (plus des troisquarts) en Moldavie et au Kosovo. Même dans les pays qui figurent tout en bas du tableau, plus de la moitié des participants en Bosnie-Herzégovine, au Monténégro et en Serbie partagent ces mêmes vues.

Aujourd'hui, la formation pédagogique sur le terrain semble être le point critique des systèmes nationaux, non seulement dans les pays des Balkans Occidentaux, en pleine transition, mais plus probablement à l'échelle de l'Europe tout entière (voir par exemple le cas de l'Autriche dans cette étude). Cependant, lorsque l'on remet en question les systèmes éducatifs de l'Europe du Sud-Est, il est important de remarquer qu'en moyenne, les enseignants en 
exercice n'accèdent que difficilement à une offre de formation continue de qualité.

Les activités sur le terrain semblent organisées et financées de manière très différente selon les pays. Il semble également qu'elles soient fréquemment relativement fragmentées. Un tiers des participants ont assisté à trois, quatre ou cinq séminaires au cours des douze derniers mois alors qu'un autre tiers n'a assisté qu'à un séminaire ou deux. Il est intéressant de souligner que quelques participants ont suivi un nombre relativement élevé de séminaires : environ un cinquième en a suivi de six à dix, voire davantage (mais ces mêmes participants n'avaient pas l'air particulièrement enthousiastes). Enfin, un dixième n'avait participé à aucune activité. Dans un cas, le Monténégro, cette proportion s'écartait de manière importante de la moyenne $(42,3 \%)$ et constituait une sorte de signal d'alarme en matière de formation continue.

Lorsqu'on leur a demandé d'évaluer les bénéfices de ces séminaires, la moitié des participants ont considéré que ces activités avaient contribué de manière importante à développer les connaissances et les compétences dont ils avaient besoin pour travailler de manière efficace dans leur établissement. Seuls quelques rares enseignants ont déclaré que ces mêmes activités n'avaient en rien amélioré leurs connaissances et leurs compétences. Ce groupe est un petit peu plus important en Bosnie-Herzégovine, alors qu'il n'est pas du tout représenté en Autriche, en Macédoine et en Slovénie. On a également demandé aux enseignants s'ils trouvaient fréquemment leur sujet favori et/ou le plus important dans l'offre actuelle de formation sur le terrain. Là encore, la moitié d'entre eux étaient relativement satisfaits de l'offre (cette réponse est la plus fréquente en Moldavie et en Serbie et la moins fréquente en Croatie et au Monténégro). Un tiers d'entre eux a émis des critiques et a déclaré ne trouver leur sujet favori que parfois ou presque jamais (réponse fréquente en Croatie, Macédoine et Bosnie-Herzégovine).

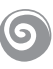

Notre étude de 2005-2006 a été la première tentative de cartographier systématiquement, dans une perspective comparative, la situation globale en matière de formation des enseignants dans la région. C'était également un projet mené dans un excellent esprit de partenariat par des chercheurs provenant de pays qui, une dizaine d'années auparavant, étaient déchirés par les conflits et par la guerre. On a également présenté les résultats de l'étude aux responsables politiques des pays de la région. Cependant, cette étude a été conduite il y a maintenant presque cinq ans et il est aujourd'hui évidemment nécessaire de lancer un nouveau projet de taille comparable. Beaucoup d'eau est passée sous les ponts au cours de ces cinq dernières années. Ce serait une bonne chose de considérer ces changements avec un regard objectif. D'autre part, la région a encore besoin de l'assistance européenne et internationale. En particulier, elle a besoin 
d'un financement pour renforcer la coopération régionale. Dans la plupart des cas, les petits systèmes nationaux tireraient le plus grand profit d'un travail commun pour atteindre des objectifs communs - qu'ils soient européens ou régionaux.

\section{BiBLIOGRAPHIE}

GABER S. (2000) : "Strategies of Educational Reform in the Countries of South Eastern Europe. Final Report.” Conclusions du séminaire (Eds. S. Gabršček and N. Dimc), Bled, Slovenia: 8-10 June 2000.

PANTIĆ N. (Ed.) (2008) : Tuning Teacher Education Curricula in the Western Balkans. Belgrade: Centre for Education Policy (CEP).

PANTIĆ N., CLOSS A. and IVOŠEVIĆ V. (2010) : Teachers for the Future. Teacher Development for Inclusive Education in the Western Balkans. Rapport soumis à l'European Training Foundation par le Centre for Education Policy [CEP, Belgrade] et par Scienter [Bologna]. Bologna, August.

ZGAGA P. (2003) : "Teachers Education and the Bologna Process. A Survey on Trends in Learning Structures at Institutions of Teachers' Education." Buchberger, F. and Berghammer S. (Eds.), Education Policy Analyses in a Comparative Perspective. Linz: Universitätsverlag Rudolf Trauner.

ZGAGA P. (2005) : The Importance of Education in Social Reconstruction. Six years of the enhanced Graz process: developments, current status and future prospects of education in South-east Europe. Ljubljana: University of Ljubljana, Centre for Educational Policy Studies.

ZGAGA P. (Ed.) (2006): The Prospects of Teacher Education in South-east Europe. Ljubljana: University of Ljubljana, Centre for Educational Policy Studies. 
